\title{
The Anti-Nociception Effect of Dezocine in a Rat Neuropathic Pain Model
}

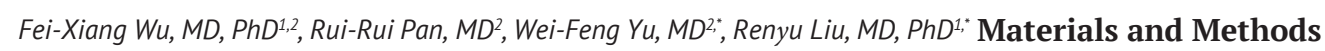

\section{Abstract}

The treatment of neuropathic pain (NP) currently remains clinically challenging. In an attempt to identify novel targets of known opioids, we found that dezocine, a non-addictive opioid, inhibits norepinephrine and serotonin reuptake through their transporter proteins which open the potential for dezocine to manage NP. In the present study, the effect of dezocine on NP was observed in a rat model of chronic constriction injury (CCI). The paw withdrawal latency $(P W L)$ and paw withdrawal threshold (PWT) were used to evaluate thermal hyperalgesia and mechanic allodynia for nociceptive response. PWL and PWT tests were performed at 11:00 AM starting from 1 day before CCI surgery and 1, 3, 7, 10 days after right sciatic nerve ligation in the presence or absence of daily intraperitoneal injection of dezocine. The results demonstrated that the CCI-induced thermal and mechanical pain hypersensitivity was attenuated by dezocine significantly and persistently without sign of tolerance, indicating that dezocine could be an alternative medication for the treatment of NP. Clinical trial to confirm such discovery is warranted.

\section{Introduction}

Neuropathic pain (NP), characterized by hyperalgesia and allodynia, is often due to nerve injuries in peripheral or central nerves system caused by trauma, inflammation or other diseases. [1] NP is affecting about $3-4.5 \%$ of the global populations and is being recognized as a major economic and social burden. [2] However, the pathophysiological mechanism of NP remains unclear and the treatment of NP is still a clinical challenge.

Opioid analgesics, such as morphine, are the widely used for various pain. But for neuropathic pain, studies have shown considerable controversy for opioid analgesics. [3] While opioids have been reported to be not effective for NP in some clinical studies $[4,5]$ and opioids have notorious side effects (constipation, nausea, vomiting and sedation).[6,7] Efforts finding opioids as alternative medication for NP manage-ment never stop. Some recent reports indicate that some types of opioids or different route of opioid administration are effective in suppressing NP.[8,9] Buprenorphine for pain management is gaining popularity in recently years due to its unique pharmacological profile as partial mu agonist and kappa antagonist and its clinical role in addiction and NP treatment. However, buprenorphine itself can generate dependence and addiction for long-term usage,[10] which is a limiting factor for its clinical use for pain management.

Similar to buprenorphine, dezocine is also a partial mu agonist and kappa antagonist.[11] Dezocine also inhibited the norepinephrine transporter (NET) and serotonin transporter (SERT).[11] As transporters, NET/SERT respectively transport norepinephrine $(\mathrm{NE}) /$ serotonin into presynaptic neurons, both of which have been suggested to be associated with NP pain. [12-14] Most importantly, dezocine is not a controlled substance despite it is an opioid and has been wildly using for post-operative pain treatment.[15] Such unique pharmacological profile of dezocine indicates that it has a potential of reducing neuropathic pain.

In the current study, we hypothesized that dezocine could attenuate neuropathic pain and demonstrated such hypothesis using a rat neuropathic pain model of chronic constriction injury (CCI) to sciatic nerve in rats.
Dezocine $(5 \mathrm{mg} / 1 \mathrm{ml}$ containing in an ampoule, Approval Number: H20080329) was obtained from Yangtze River Pharmaceutical Group (Jiangsu, China). Sodium pentobarbital was obtained from Chemical Reagent Company (Shanghai, China).

\section{Animals}

The National Institute of Health guidelines for Ethical Conduct in the Care and Use of Animals were strictly followed the experimental protocol approved by the institutional review Committee of Experimental Animal Care. Male Sprague-Dawley rats (Age: 10-12week, Weight: 200-250g, from Shanghai Experimental Animal Center of Chinese Academy of Sciences) were housed in a specific pathogen free (SPF) environment with a 12/12 hour light /dark cycle.

\section{Chronic constriction injury (CCI) model}

CCI procedures on the sciatic nerve of male SD rats were performed as previously described.[16] Briefly, after rats were anesthetized by i.p. injection of sodium pentobarbital $(40 \mathrm{mg} / \mathrm{kg})$, the right sciatic nerve of the mid-thigh level was exposed. Chromic gut 4-0 was loosely tied around the nerve for 4 ligatures with about $1 \mathrm{~mm}$ between knots. The ligation was performed to just barely reduce the diameter of sciatic nerve. The ligatures caused intraneural edema and resulted in constriction of nerve. In the sham group, the sciatic nerve was exposed without ligation. The incisions of rats were closed in layers. After recovery from anesthesia, rats were housed individually in the clear plastic cages with soft bedding covered with 3-6 cm of sawdust.

\section{Experimental Protocol}

Rats were randomly assigned to three groups (6 rats in each group): a sham group (IP normal saline, IP NS), an NS group (CCI+ IP NS) and a Dezocine group (CCI+ IP dezocine). In the dezocine group, rats of CCI model received intraperitoneal (IP) injection of $3 \mathrm{mg} / \mathrm{kg}$ (in $2 \mathrm{ml}$ of volume) body weight of dezocine at 9:00 AM per day starting for the day of the surgery. Same volume of normal saline $(2 \mathrm{ml})$ was injected in the other two groups at the same time.

\section{Evaluation of thermal hyperalgesia}

The paw withdrawal latency (PWL) to radiant heat was used to evaluate thermal hyperalgesia for nociceptive response as previously described.[17] Rats were placed in transparent plexiglass cage $(23 \times 18 \times 13 \mathrm{~cm})$ with a piece of 3 -mm-thick glass floor and received heat radiation after acclimating to the environments for 30 minutes. The radiant heat source consists of a high-intensity projection lamp bulb $(8 \mathrm{~V}, 50 \mathrm{~W})$, which was located $40 \mathrm{~mm}$ below the glass floor beneath the right hind paw of the rats. The heat source projected through a $5 \times 10$-mm aperture on the top of a movable case. A digital timer automatically measured the duration between the starting of heat and the paw withdrawal, which was considered as the PWL. The PWL was measured in 0.1 second and a maximum of 20 seconds exposure to radiation was set to avoid injury. Three repeated measurements were performed in each rat with a 5-minute interval between each measurement. 
PWL tests were performed at 11:00 AM starting from 1 day before CCI surgery and 1, 3, 7, 10 days after surgery.

\section{Evaluation of tactile allodynia}

The paw withdrawal threshold (PWT) was used to evaluate mechanical allodynia for nociceptive response with Von Frey filaments. The rats were placed in transparent plexiglass cage with a wire mesh floor. After acclimating to their environments for 30 minutes, each filament was applied perpendicularly to the plantar surface of the right hind paw. The end point was determined as paw withdrawal accompanied by biting, head turning and/or licking. The force (in gram, g) needed for this reaction was recorded. [18] The PWT was taken though increasing and decreasing the stimulus strength sequentially with the 'up-and-down' method as described by Chaplan.[19] Similar to PWL test, PWT tests were performed at 1 day before and 1, 3, 7, 10 days after CCI surgery.

\section{Statistical analysis}

All data were presented as mean \pm SEM. Statistical analysis was performed using two-way ANOVA via GraphPad Prism5 software (GraphPad Software Inc, CA, USA). P $<0.05$ was considered statistically significant.

\section{Results}

After surgery, the PWL, representing the threshold of thermal hyperalgesia, decreased significantly compared to sham group. Statistically significant difference was found between the NS group and the sham group on 1, 3, 7, 10 days after CCI surgery $(\mathrm{P}<0.05$, figure 1). Comparing to NS group, after dezocine administration, PWL significantly improved in the dezocine group lasting for 10 days without signs of fluctuation $(P<0.05)$, suggesting that dezocine could attenuate thermal hyperalgesia during the whole experimental period without signs of tolerance.

PWT was utilized to measure mechanical allodynia. Mechanical allodynia was induced by CCI, as evidenced by the reduction of PWT (figure 2). CCI rats receiving intraperitoneal injection of dezocine, PWT was increased markedly in the dezocine group comparing to the NS group $(\mathrm{P}<0.05)$, which suggested an attenuation of allodynia by dezocine (figure 2). Similar to PWL, the improvement of PWT was found during the entire experiment period. Taken together, the anti-nocicetion effect by dizocine started immediately after administration lasted for 10 days without signs of tolerance.

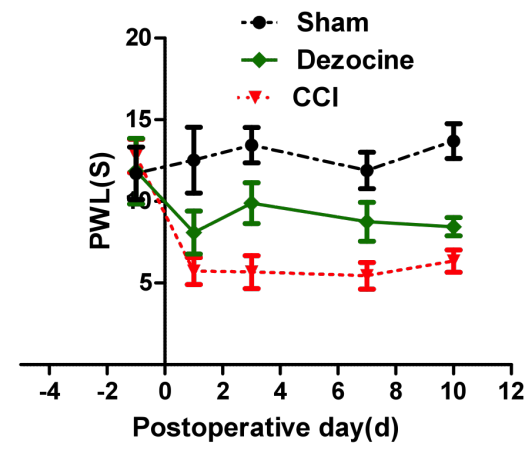

Fig.1 Changes of PWL after injection of dezocine on CCI rats. Rats were administered with dezocine one day before CCI, and then PWL was measured. Following administration of dezocine, PWL was significantly increased comparing to that in the NS group $\left({ }^{*} \mathrm{P}<0.05\right)$.

\section{Discussion}

In the present study, the effect of dezocine on NP was investigated in a rat CCI model. The results indicated that dezocine significantly attenuated the CCI-induced thermal and mechan-

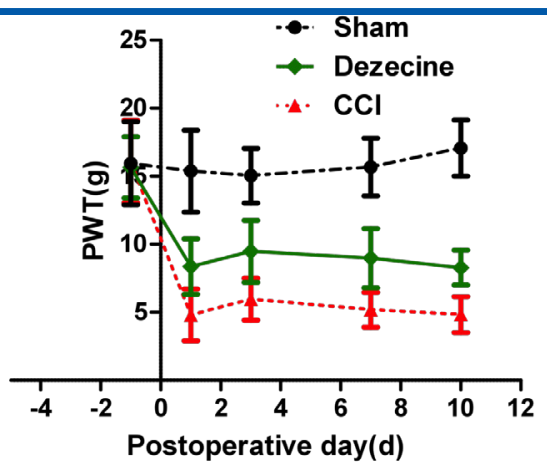

Fig. 1 Changes of PWT after injection of dezocine on CCI rats. PWT was monitored one day before CCI and 1, 3, 7, and 10 day after surgery. At 1st, 3rd 7th and 10th day, PWT showed markedly increased comparing to that in the NS group $\left({ }^{*} \mathrm{P}<0.05\right)$.

ical pain hypersensitivity, indicating that dezocine could be an alternative medication for the treatment of NP. While this is a rather simple study, the novel clinical implication for dezocine is enormous due to lack to good medication for NP management.

Dezocine is a non-addiction analgesic drug with partial mu-opioid agonist and kappa-opioid antagonist activity,[11,20] which was used for alleviating post-operative pain.[21] The analgesic effect is approximately equipotent with morphine after administration of dezocine with therapeutic doses for moderate to severe pain.[22] The same analgesic effect of dezocine could reach that of fentanyl for postoperative pain treatment.[23] Partial mu-opioid agonist generally has fewer the side effects such as respiratory depression, tolerance, drug dependence and pruritus, than that of full mu-opioid agonist.[24,25] Tolerance and addiction serve as major barriers for the long-term usage of opioids for neuropathic pain especially for full mu-opioid agonist like morphine.[26] In the present study, no significant tolerance is observed for dezocine after intermittent injection of dezocine for consecutive 10 days. Tolerance generally develops after 7 days of morphine administration in rats.[27,28] Further studies are needed to confirm the finding of lack of tolerance for longer time period of usage.

While the mechanism of effectiveness of dezocine in reducing NP, it is possible that such therapeutic effectiveness may be related to the novel targets of dezocine we identified recently. In addition to opioid receptors, both NET and SERT were identified as novel targets of dezocine in our recent work using molecular target profiling. Dezocine inhibited norepinephrine and serotonin reuptake in a concentration-dependent manner in vitro. Through norepinephrine reuptake, NET plays a role in regulating the concentration of NE which is an important factor in descending inhibitory pathway.[29] In NP models of L5-L6 spinal nerve ligation, the increasing of NET density in the lumbar spinal cord was found, suggesting NET as a target for NP treatment.[30] Various studies have demonstrated the analgesic actions of NP through norepinephrine reuptake inhibitors.[31,32] SERT has proven to be involved in NP and serotonin reuptake inhibitors have indicated to be effective for NP treatment through inhibition of SERT. $[14,33]$ It is important to know that many drugs such as duloxetine, amitriptyline, and Thien-2-yl 1S, 2R-milnacipran analogues, are all mixed NET/SERT re-uptake inhibitors, which have been shown efficacy against NP in different animal models and in clinical use.[34-37] In our present study, the attenuation effect of allodynia and hyperalgesia in NP model by intraperitoneal injection of dezocine was found. Based on the role of NET and SERT in NP and our previous founding that NET and SERT are new targets of dezocine, we assume that the anti-nociception effect of dezocine may through the inhibition of norepinephrine and 
serotonin reuptake. Thus, dezocine may be a good choice of NP treatment through opioid system and norepinephrine/serotonin system. Further studies are needed to confirm related mechanism and demonstrate the effectiveness in clinical trials.

\section{Conclusion}

In summary, dezocine significantly attenuated the nociception effect in a neuropathic pain model in rats; indicating that dezocine could be an alternative medication for neuropathic pain management.

\section{Address for correspondence}

Corresponding author: Renyu Liu, M.D., Ph.D. Assistant Professor. Department of Anesthesiology and Critical Care, Perelman School of Medicine at the University of Pennsylvania

Address: 336 John Morgan building, 3620 Hamilton Walk, Philadelphia PA, 19104 USA.

Phone: 2156623750

Fax: 2153495078

Email: liur@uphs.upenn.edu

Weifeng Wu, MD, PhD, Professor and Chair, Department of Anesthesia \& Intensive Care, Eastern Hepatobiliary Surgical Hospital Address: The Second Military Medical University, Shanghai, 200438, China. Phone: 86-21-55063846

Fax: 86-21-25070783

Email:ywf808@sohu.com

\section{Disclosure of Funding}

NIH K08 (K08-GM-093115) (PI, RL) and the Department of Anesthesiology and Critical Care at the University of Pennsylvania (PI, RL).

\section{Additional publication details}

Journal short name: Trans Periop \& Pain

MedReceived Date:May 1, 2014

Accepted Date: June 15, 2014

Published Date: July 1, 2014

\section{Citation and Copyrights}

Citation: Fei-Xiang Wu, Rui-Rui Pan, Wei-Feng Yu, Renyu Liu. Dezocine and neuropathic pain. Trans Periop \& Pain Med 2014, 1(1):5-8

Copyright: This is an open-access article distributed under the terms of the Creative Commons Attribution License, which permits unrestricted use, distribution, and reproduction in any medium, provided the original author and source are credited.

References

1. Liou JT, Lee CM, Day YJ (2013) The immune aspect in neuropathic pain: Role of chemokines. Acta Anaesthesiol Taiwan 51: 127-132.

2. del Rey A, Apkarian AV, Martina M, Besedovsky HO (2012) Chronic neuropathic pain-like behavior and brain-borne IL-1beta. Ann N Y Acad Sci 1262: 101-107.

3. Moulin DE, Clark AJ, Gilron I, Ware MA, Watson CP, et al. (2007) Pharmacological management of chronic neuropathic pain - consensus statement and guidelines from the Canadian Pain Society. Pain Res Manag 12: 13-21.
4. Arner S, Meyerson BA (1988) Lack of analgesic effect of opioids on neuropathic and idiopathic forms of pain. Pain 33: 11-23.

5. Choi JI, Kim WM, Yoon MH, Lee HG (2010) Antiallodynic Effect of Thalidomide and Morphine on Rat Spinal Nerve Ligation-induced Neu-ropathic Pain. Korean J Pain 23: 172-178.

6. Viscusi ER, Frenkl TL, Hartrick CT, Rawal N, Kehlet H, et al. (2012) Perioperative use of etoricoxib reduces pain and opioid side-effects after total abdominal hysterectomy: a double-blind, randomized, placebo-controlled phase III study. Curr Med Res Opin 28: 1323-1335.

7. Du BX, Song ZM, Wang K, Zhang H, Xu FY, et al. (2013) Butorphanol prevents morphine-induced pruritus without increasing pain and other side effects: a systematic review of randomized controlled trials. Can J Anaesth 60: 907-917.

8. Smith HS (2012) Opioids and neuropathic pain. Pain Physician 15: ES93-110.

9. Wu WT, Huang YH, Chen DC, Chou LW (2013) Effective management of intractable neuropathic pain using an intrathecal morphine pump in a patient with acute transverse myelitis. Neuropsychiatr Dis Treat 9: 1023-1028.

10. Coluzzi F, Pappagallo M (2005) Opioid therapy for chronic noncancer pain: practice guidelines for initiation and maintenance of therapy. Minerva Anestesiol 71:425-433.

11. Liu R, Huang X-P, Yeliseev A, Xi J, Roth BL (2014) Novel molecular targets of dezocine and their clinical implications. Anesthesiology 120: 714-723.

12. Hartrick CT (2012) Noradrenergic reuptake inhibition in the treatment of pain. Expert Opin Investig Drugs 21: 1827-1834.

13. Sindrup SH, Gram LF, Brosen K, Eshoj O, Mogensen EF (1990) The selective serotonin reuptake inhibitor paroxetine is effective in the treatment of diabetic neuropathy symptoms. Pain 42: 135-144.

14. Vogel C, Mossner R, Gerlach M, Heinemann T, Murphy DL, et al. (2003) Absence of thermal hyperalgesia in serotonin transporterdeficient mice. J Neurosci 23: 708-715.

15. Zhu Y, Jing G, Yuan W (2011) Preoperative administration of intramuscular dezocine reduces postoperative pain for laparoscopic cho-lecystectomy. J Biomed Res 25: 356-361.

16. Bennett GJ, Xie YK (1988) A peripheral mononeuropathy in rat that produces disorders of pain sensation like those seen in man. Pain 33: 87-107.

17. Hargreaves K, Dubner R, Brown F, Flores C, Joris J (1988) A new and sensitive method for measuring thermal nociception in cutaneous hyperalgesia. Pain 32: 77-88.

18. Wu JX, Xu MY, Miao XR, Lu ZJ, Yuan XM, et al. (2012) Functional up-regulation of $\mathrm{P} 2 \mathrm{X} 3$ receptors in dorsal root ganglion in a rat model of bone cancer pain. Eur J Pain 16: 1378-1388.

19. Chaplan SR, Bach FW, Pogrel JW, Chung JM, Yaksh TL (1994) Quantitative assessment of tactile allodynia in the rat paw. J Neurosci Methods 53: 55-63.

20. Barrett AC, Smith ES, Picker MJ (2003) Capsaicin-induced hyperalgesia and mu-opioid-induced antihyperalgesia in male and female Fischer 344 rats. J Pharmacol Exp Ther 307: 237-245. 
21. Cohen RI, Edwards WT, Kezer EA, Ferrari DA, Liland AE, et al. (1993) Serial intravenous doses of dezocine, morphine, and nalbuphine in the management of postoperative pain for outpatients. Anesth Analg $77: 533-539$.

22. Walker EA, Tiano MJ, Benyas SI, Dykstra LA, Picker MJ (1999) Naltrexone and beta-funaltrexamine antagonism of the antinociceptive and response rate-decreasing effects of morphine, dezocine, and d-propoxyphene. Psychopharmacology 144: 45-53.

23. Anderson D (2011) A review of systemic opioids commonly used for labor pain relief. J Midwifery Womens Health 56: 222-239.

24. Gharagozlou P, Demirci H, David Clark J, Lameh J (2003) Activity of opioid ligands in cells expressing cloned mu opioid receptors.

BMC Pharmacol 3: 1 .

25. Smith H, Bruckenthal P (2010) Implications of opioid analgesia for medically complicated patients. Drugs Aging 27:417-433.

26. Mayer DJ, Mao J, Price DD (1995) The association of neuropathic pain, morphine tolerance and dependence, and the translocation of protein kinase C. NIDA Res Monogr 147: 269-298.

27. Kong Q, Yu LC (2013) Antinociceptive effects induced by intra-periaqueductal grey injection of the galanin receptor 1 agonist M617 in rats with morphine tolerance. Neurosci Lett 550: 125-128.

28. Jun IG, Kim SH, Yoon YI, Park JY (2013) Intrathecal lamotrigine attenuates antinociceptive morphine tolerance and suppresses spinal glial cell activation in morphine-tolerant rats. J Korean Med Sci 28: 300-307.

29. Obara G, Toyohira Y, Inagaki H, Takahashi K, Horishita T, et al. (2013) Pentazocine inhibits norepinephrine transporter function by reducing its surface expression in bovine adrenal medullary cells.J Pharmacol Sci 121: 138-147.

30. Rojo ML, Rodriguez-Gaztelumendi A, Pazos A, Diaz A (2012) Differential adaptive changes on serotonin and noradrenaline transport-ers in a rat model of peripheral neuropathic pain. Neurosci Lett 515: 181-186.

31. Jefferies K (2010) Treatment of neuropathic pain. Semin Neurol 30: 425-432.

32. Farghaly HS, Abdel-Zaher AO, Mostafa MG, Kotb HI (2012) Comparative evaluation of the effect of tricyclic antidepressants on inducible nitric oxide synthase expression in neuropathic pain model. Nitric Oxide 27: 88-94.

33. Sindrup SH, Bjerre U, Dejgaard A, Brosen K, Aaes-Jorgensen T, et al. (1992) The selective serotonin reuptake inhibitor citalopram relieves the symptoms of diabetic neuropathy. Clin Pharmacol Ther 52: 547-552.

34. Whiteside GT, Dwyer JM, Harrison JE, Beyer CE, Cummons T, et al. (2010) WAY-318068: a novel, potent and selective noradrenaline reuptake inhibitor with activity in rodent models of pain and depression. $\mathrm{Br}$ J Pharmacol 160: 1105-1118.

35. Dyck B, Tamiya J, Jovic F, Pick RR, Bradbury MJ, et al. (2008) Characterization of thien-2-yl 1S,2R-milnacipran analogues as potent norepinephrine/serotonin transporter inhibitors for the treatment of neuropathic pain. J Med Chem 51: 7265-7272.

36. Tanenberg RJ, Clemow DB, Giaconia JM, Risser RC (2013) Duloxetine Compared with Pregabalin for Diabetic Peripheral Neuropathic Pain Management in Patients with Suboptimal Pain Response to Gabapentin and Treated with or without Antidepressants: A Post Hoc Analysis. Pain Pract doi: 10.1111/papr.12121.

37. Banerjee M, Pal S, Bhattacharya B, Ghosh B, Mondal S, et al.
(2013) A comparative study of efficacy and safety of gabapentin versus amitriptyline as coanalgesics in patients receiving opioid analgesics for neuropathic pain in malignancy. . Indian J Pharmacol 45: 334-338. 